\title{
Avances en el estudio fractográfico de fibras cerámicas de circona- erbia mediante microscopía óptica confocal
}

\author{
J. M. LÓPEZ-CEPERO, J. J. QUISPE CANCAPA, A. R. DE ARELLANO LÓPEZ, J. MARTÍNEZ FERNÁNDEZ \\ Departamento de Física de la Materia Condensada, Universidad de Sevilla. \\ Apdo. 1065, 41080 - Sevilla
}

\begin{abstract}
La microscopía óptica confocal (LSCM, Laser Scanning Confocal Microscopy) es una técnica microscópica basada en una construcción óptica que permite eliminar la luz procedente de zonas no enfocadas de la muestra. Mientras que es de amplio uso en Ciencias de la Vida, la aplicación de LSCM a la Ciencia de Materiales no ha sido apenas explorada, siendo prácticamente inexistentes los estudios fractográficos que se apoyen en LSCM. A pesar de ello, sus características (obtención de información tridimensional, resolución por debajo de la micra y sencilla preparación de muestras) la convierten en una herramienta idónea para una multitud de problemas fractográficos, debido a la obtención rápida de valiosa información y a su buena coordinación con la microscopía electrónica de barrido (SEM).

En este trabajo, los autores estudian en detalle un sistema de interés (fibras cerámicas de circona dopada con un 5\% molar de erbia, sometidas a ensayos de tracción a alta temperatura) mediante LSCM. Además de poner de relieve la utilidad de la técnica y de revelar la textura característica de la superficie de fractura de dichas fibras, se encuentra que dicha textura refleja la estructura nanométrica de precipitados propia del material.
\end{abstract}

Palabras clave: LSCM, confocal, fractografía, circona, circona-erbia.

Advances in fractographical studies of ceramic erbia-doped zirconia fibers using laser scanning confocal microscopy

Laser scanning confocal microscopy (LSCM) is a microscopic technique based on an optical construction which allows the microscope to discard the light coming from unfocused zones of the sample. Whereas LSCM is extensively used in Natural Sciences (Biology, Medicine...), its use in Materials Science is almost unexplored and, in particular, there are essentially no fractographical studies using LSCM. However, its characteristics (gathering of 3D information, better than micron resolution and simple sample preparation) make LSCM an ideal tool in a wide selection of fractographical problems, owing to the fast adquisition of valuable information and to the excellent sinergy with scanning electron microscopy (SEM).

In the present work, the authors study an interesting system $\left(\mathrm{ZrO}_{2}-5 \% \mathrm{~mol} \mathrm{Er}_{2} \mathrm{O}_{3}\right.$ fibers, submitted to tensile strength in hightemperature conditions) in detail with LSCM. In addition to showcasing the usefulness of LSCM in fractographical studies and revealing the characteristic texture of the fracture surface in such fibers, said texture is found to closely resemble the nanometric precipitate structure which is unique to this material.

Keywords: LSCM, confocal, fractograpy, zirconia, erbia-doped zirconia.

\section{INTRODUCCIÓN}

Si bien la microscopía óptica confocal de barrido láser (LSCM) fue originalmente desarrollada para aplicaciones en Ciencias de la Vida, tales como Farmacia, Medicina o Biología, recientemente ha venido cobrando interés la extensión de esta versátil técnica a otros campos del conocimiento, entre los que destaca de forma específica la Ciencia de Materiales (1). Debido a que LSCM permite la obtención de información tridimensional acerca de la superficie observada, los resultados obtenidos por LSCM son en muchas ocasiones imposibles de obtener mediante técnicas más tradicionales, como la microscopía electrónica de barrido (SEM). En concreto, los autores han demostrado en trabajos anteriores $(2,3,4)$ la viabilidad de LSCM como herramienta fractográfica, aplicación aún poco explorada pero que muestra resultados muy prometedores, fundamentados esencialmente en lo adecuado de las características de LSCM para abordar el problema (resolución lateral y en alturas, rapidez de adquisición de los datos, trivial preparación de muestras y muy buena coordinación con SEM).

Tras los estudios preliminares mencionados, resulta natural continuar con el trabajo estudiando más en profundidad algún tipo concreto de material. El objetivo es doble: en primer lugar, y desde el punto de vista del desarrollo de la técnica, ayuda a establecer la utilidad de LSCM como herramienta fractográfica para el material particular, permitiendo el desarrollo de protocolos específicos que extraigan el máximo partido a la técnica; en segundo lugar, y desde un punto de vista más fundamental, el centrarse en un problema y comparar los resultados obtenidos con los provenientes de técnicas más tradicionales (microscopía electrónica, por ejemplo) permite encontrar los puntos fuertes de la técnica y obtener resultados novedosos, así como establecer las posibles sinergias entre LSCM y el resto de técnicas fractográficas.

En el presente trabajo se estudian fibras de circona dopada con erbia sometidas a tracción en modo I en condiciones de alta temperatura $(4,5)$. Las superficies de fractura obtenidas se 
observaron mediante LSCM, obteniendo una vista global, una o varias vistas de detalle y un buen número de perfiles, con el objetivo de aislar e ilustrar las características fractográficas típicas del sistema.

\section{PROCEDIMIENTO EXPERIMENTAL}

Se emplearon fibras de circona-erbia $\left(\mathrm{ZrO}_{2}-5 \% \mathrm{~mol} \mathrm{Er}_{2} \mathrm{O}_{3}\right)$, sometidas a tensión en condiciones de alta temperatura $\left(1400^{\circ} \mathrm{C}\right)$ hasta lograr su rotura en modo I. Los ensayos de rotura, así como una exhaustiva caracterización fractográfica en SEM de la superficie de las fibras, se realizaron en el marco de una tesis doctoral sobre el tema (3) y han dado lugar a varias publicaciones $(5,6)$. Las muestras preparadas para SEM no requieren de preparación posterior para su observación en LSCM.

El método de trabajo seguido se encuentra descrito exhaustivamente en (3). Esquemáticamente, las muestras se fijan a un portaobjetos adecuado para su observación en el microscopio y se observan empleando un objetivo de 20x (NA 0.5). Se obtienen del orden de 100 imágenes, cada una de ellas a una cierta altura, siendo la diferencia de alturas entre las imágenes adquiridas del orden de las 0.4 micras; debido a la construcción óptica del microscopio confocal, la luminosidad de un punto en una de estas imágenes es máxima cuando la altura a la que se realiza la adquisición es muy cercana a la altura real del punto y decae al alejarse de ella, lo que permite obtener la altura a la que se encuentra el punto. Finalmente, el software analiza las imágenes y genera un mapa de alturas aprovechando este hecho. Dicho mapa de alturas puede elevarse, obteniendo así una vista tridimensional en perspectiva, o bien usarse como origen para obtener perfiles (cortes) de la superficie de fractura.

\section{RESULTADOS Y DISCUSIÓN}

Se presentan los resultados obtenidos para dos fibras de circona-erbia representativas. En cada caso, se muestra una vista global en planta de la fibra, junto a una vista en perspectiva y la correspondiente imagen obtenida mediante SEM, para comparar los resultados con ambas técnicas (figuras 1 y 2). A continuación, se observan una serie de detalles (uno en el caso de la primera fibra, figura 3; dos en el caso de la segunda, figura 4) a mayor aumento, de los cuales se extraen varios perfiles.

Las fibras analizadas presentan una serie de estructuras típicas que pueden ser identificadas en la superficie de fractura de prácticamente todas las fibras del material ensayadas en las mismas condiciones. Por este motivo, tienen un alto valor ilustrativo y demuestran a la perfección el tipo de conocimiento fractográfico que puede obtenerse con facilidad mediante LSCM. Además, la comparación con las imágenes SEM de las mismas fibras permite identificar las ventajas de cada una de las técnicas y poner de relieve su complementariedad.

Observando ambas tomas globales (figuras 1a y 2a), salta inmediatamente a la vista la aparición de una característica típica, que puede ser descrita como un conjunto de líneas aproximadamente paralelas que apuntan al defecto desde el que se originó la fractura, situado en la superficie de la fibra (abajo en la figura 1a, a la izquierda en la figura 2a), superpuesto al cual hay otro conjunto de líneas similares, cruzándose ambos a un ángulo próximo al recto. Por este
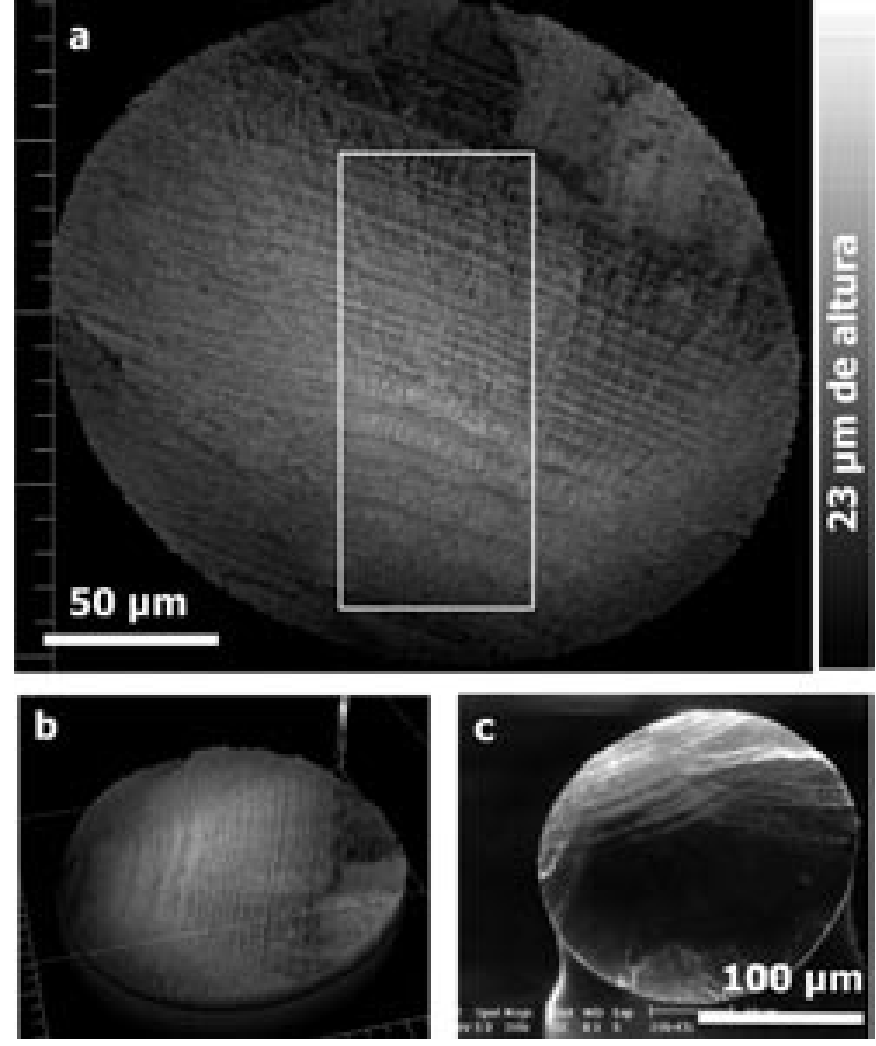

Fig. 1.- Vista de una fibra de circona-erbia típica mediante microscopía óptica confocal. Se dispone de una vista superior de la misma (a), de una vista en perspectiva (b) y de una imagen en SEM de la misma fibra a efectos de comparación (c).

motivo, una textura en forma de cuadrícula o panal cuadrado aparece en la superficie de fractura. Este hecho puede verse con gran claridad en la primera de las fibras (figura 1), aunque también está presente de forma menos evidente en la segunda de ellas (figura 2).

Se observa también que la estructura de la superficie evoluciona conforme avanza la fractura; en las zonas cercanas al defecto las diferencias en altura son menos importantes, mientras que conforme la fractura se acerca al extremo opuesto de la fibra comienza a observarse una mayor irregularidad y rugosidad. Esto puede verse de forma más clara en las vistas en perspectiva (figuras $1 \mathrm{~b}$ y $2 \mathrm{~b}$ ), donde la escala en alturas y en planta es la misma. Finalmente, la comparación con los resultados en SEM (figuras 1c y 2c) nos indica la utilidad de la técnica; las imágenes son inmediatamente identificables, ya que son similares a grandes rasgos. A pesar de la mayor resolución lateral del SEM, muchos de los detalles más sutiles, cuyo contraste está basado en una diferencia de altura, se observan de forma más clara mediante LSCM.

Centrándonos ya en la primera de las fibras, el conjunto de líneas más prominentes son aquellas que van en horizontal, mientras que las líneas que apuntan al defecto, que van en la dirección vertical, son menos evidentes pero aún claramente visibles. Esto puede observarse con mayor comodidad si nos centramos en la parte central destacada en la figura 1a, ampliada en la figura 3a; la cuadrícula de la que se habló anteriormente es visible de forma evidente, y sus características (dos conjuntos de líneas paralelas y ortogonales entre sí) se conservan de forma notable en toda la zona destacada, a 

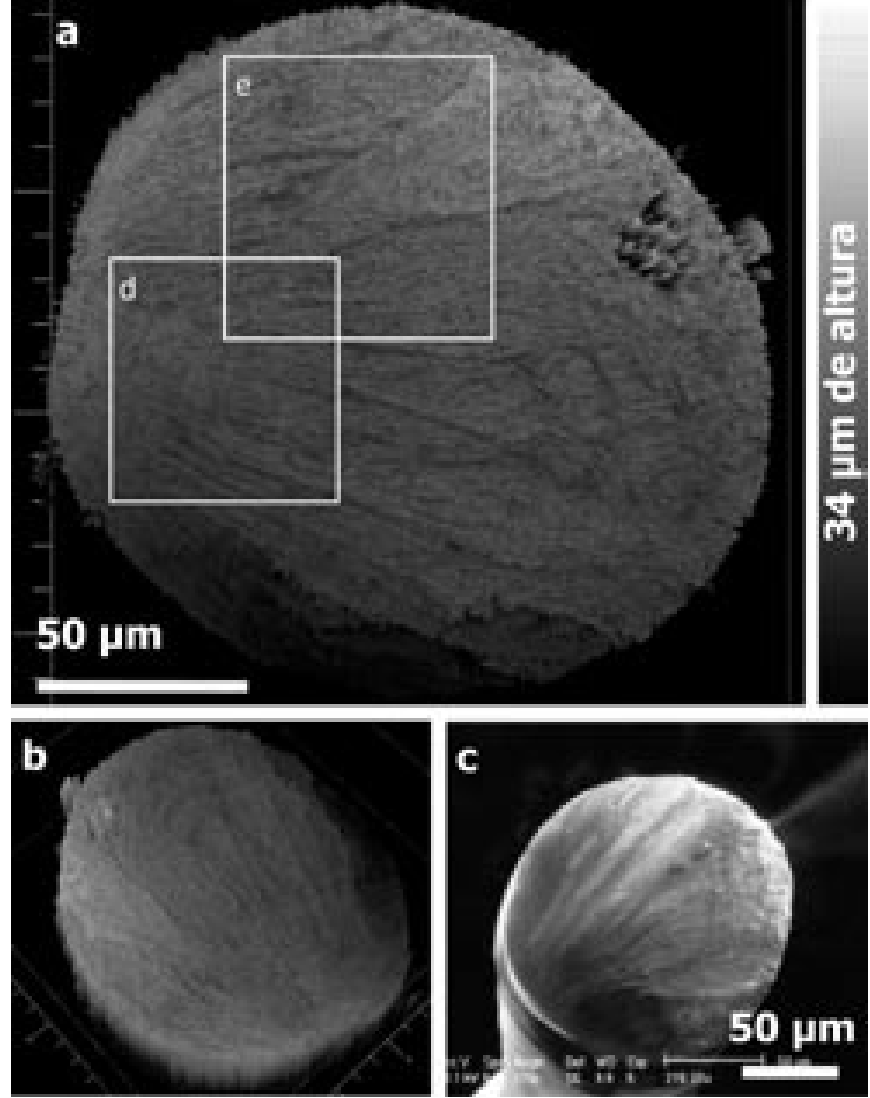

Fig. 2.- Vista de una segunda fibra de circona-erbia típica mediante microscopía óptica confocal. De nuevo se dispone de una vista superior de la misma (a), de una vista en perspectiva (b) y de una imagen en SEM de la misma (c). pesar de que abarca una buena parte del diámetro de la fibra. Junto a la vista destacada, aparecen varios perfiles (figuras $3 b$ a 3e; las flechas en la figura 3a corresponden al eje $\mathrm{X}$ de los perfiles, de izquierda a derecha), en los que se ven en detalle las sucesiones de crestas y valles que conforman las líneas. De ellos pueden extraerse dos conclusiones: por un lado, el hecho de que las líneas verticales $(3 b, 3 c)$ y las horizontales $(3 d, 3 e)$ tienen una estructura prácticamente idéntica, solo que con una profundidad característica de los valles algo menor en el caso de las primeras; y, en segundo lugar, confirma que las características de la rejilla son muy similares en zonas alejadas entre sí.

La segunda de las fibras (figura 2) muestra estructuras más complejas, por lo que la rejilla no es tan evidente. Sin embargo, la observación del mapa de alturas (figura 2a), así como de las vistas en perspectiva y SEM (2b, 2c), permite identificar una estructura muy parecida a la de la primera fibra. En este caso, las líneas mejor visibles son las que apuntan al defecto originario, que se encuentra en el borde izquierdo de la fibra, pero aparecen con dos orientaciones preferentes, ambas fundamentalmente horizontales pero una abriéndose hacia abajo (recuadro d) y otra hacia arriba (parte inferior del recuadro e). El relieve de los recuadros $2 \mathrm{~d}$ y $2 \mathrm{e}$ puede observarse con mayor detalle en las figuras $4 a$ y $4 b$, respectivamente.

La confluencia de ambos grupos de líneas da lugar a un comportamiento muy rico y complejo en la zona cercana al defecto. No obstante, cada uno de estos conjuntos presenta por separado características similares a las de la primera de las fibras, dando lugar a una estructura de crestas y valles muy parecida en todos los casos. Esto puede verse en los perfiles de la figura $4 \mathrm{a}$; los perfiles cruzados (4c y $4 \mathrm{~d}$; $4 \mathrm{f}$ y $4 \mathrm{~g}$ ) muestran un comportamiento localmente muy parecido a los de la
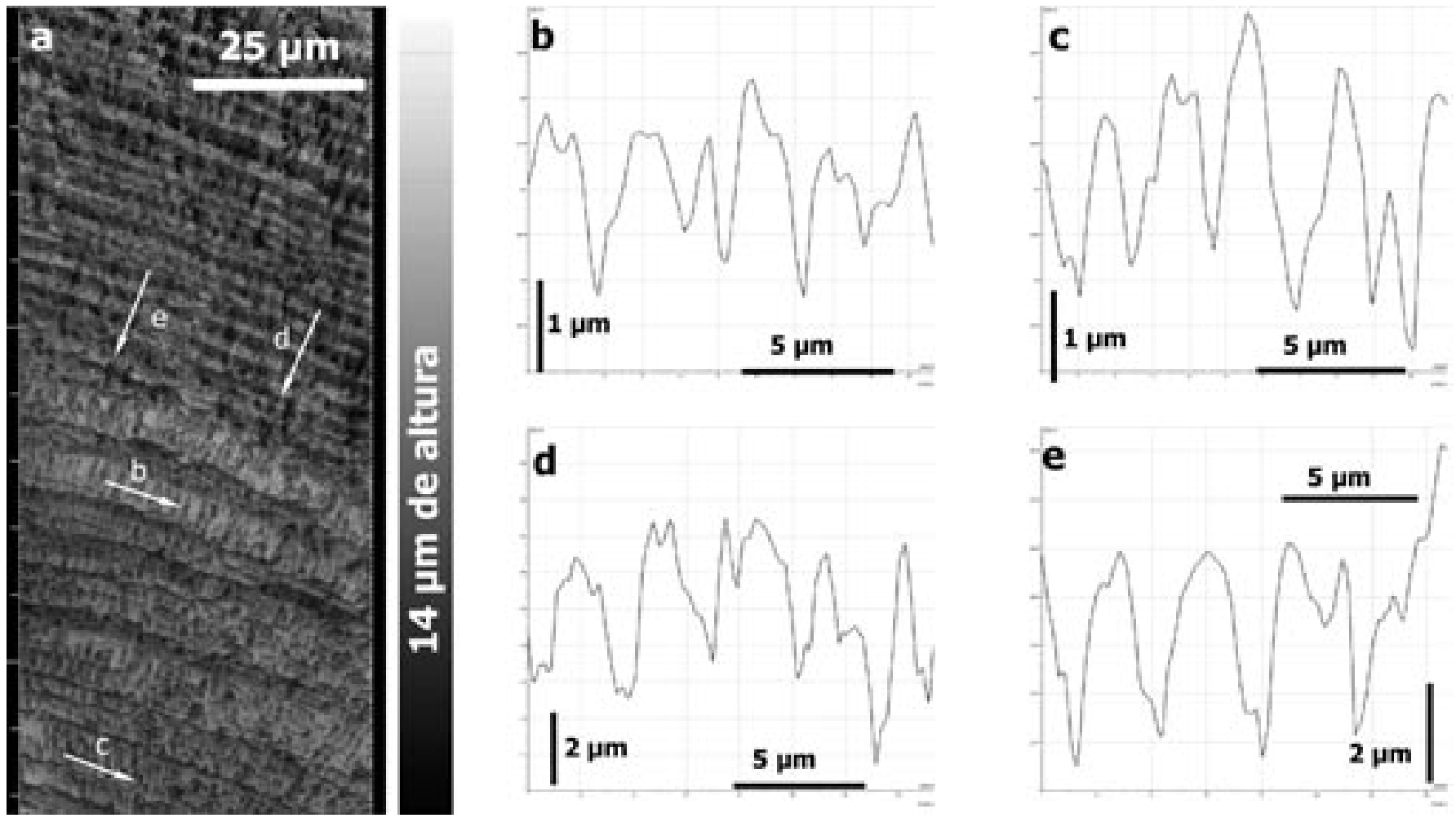

Fig. 3.- Vista de la zona recuadrada en la figura 1a. Una imagen ampliada se encuentra en (a), mientras que las gráficas (b) a (e) son perfiles asociados a los diversos cortes marcados en la misma. 

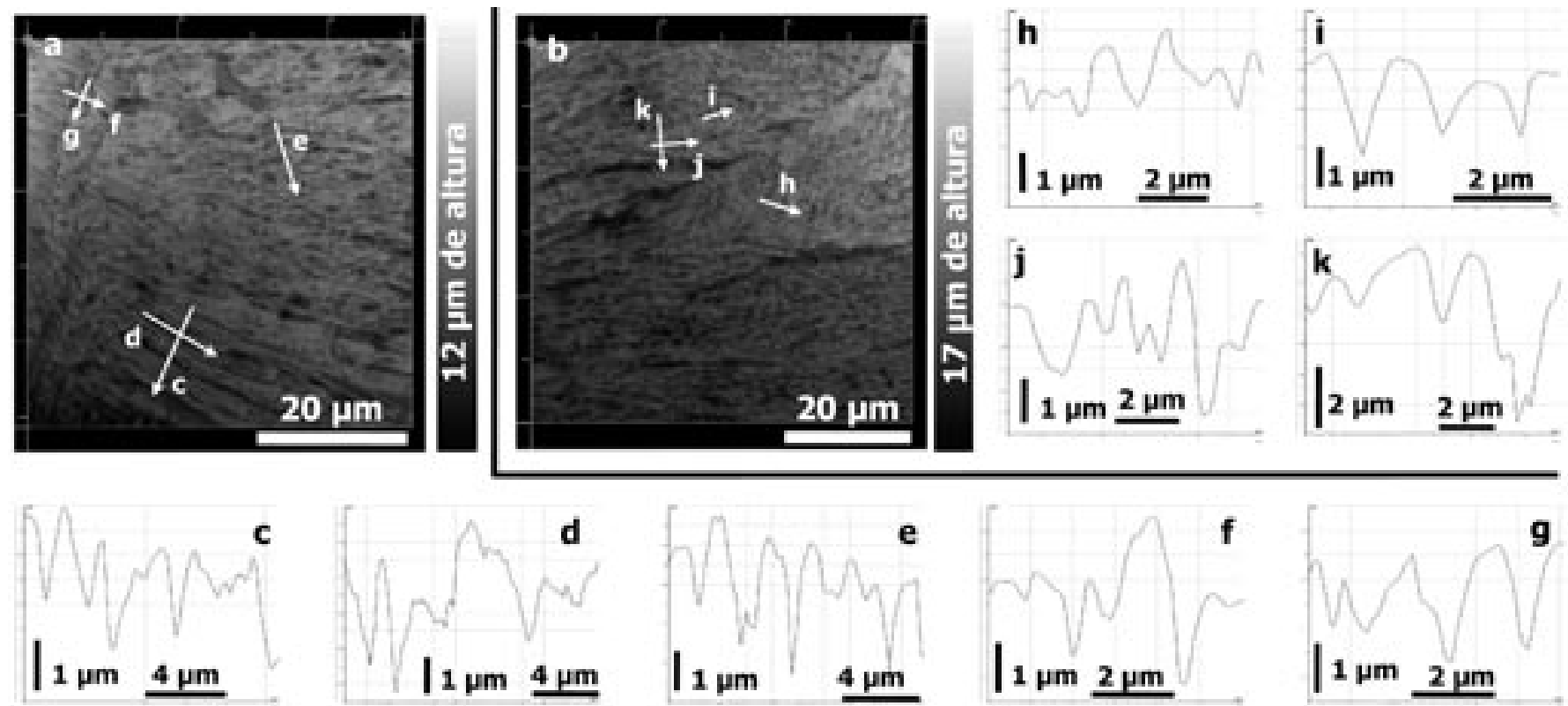

Fig. 4.- Vistas en detalle y perfiles de las zonas recuadradas en la figura 2a. La zona marcada como (a), correspondiente al recuadro $2 \mathrm{~d}$, tiene asociados los perfiles (c) a (g), mientras que la (b), correspondiente al recuadro $2 \mathrm{e}$, tiene asociados los perfiles $(\mathrm{h}) \mathrm{a}(\mathrm{k})$.
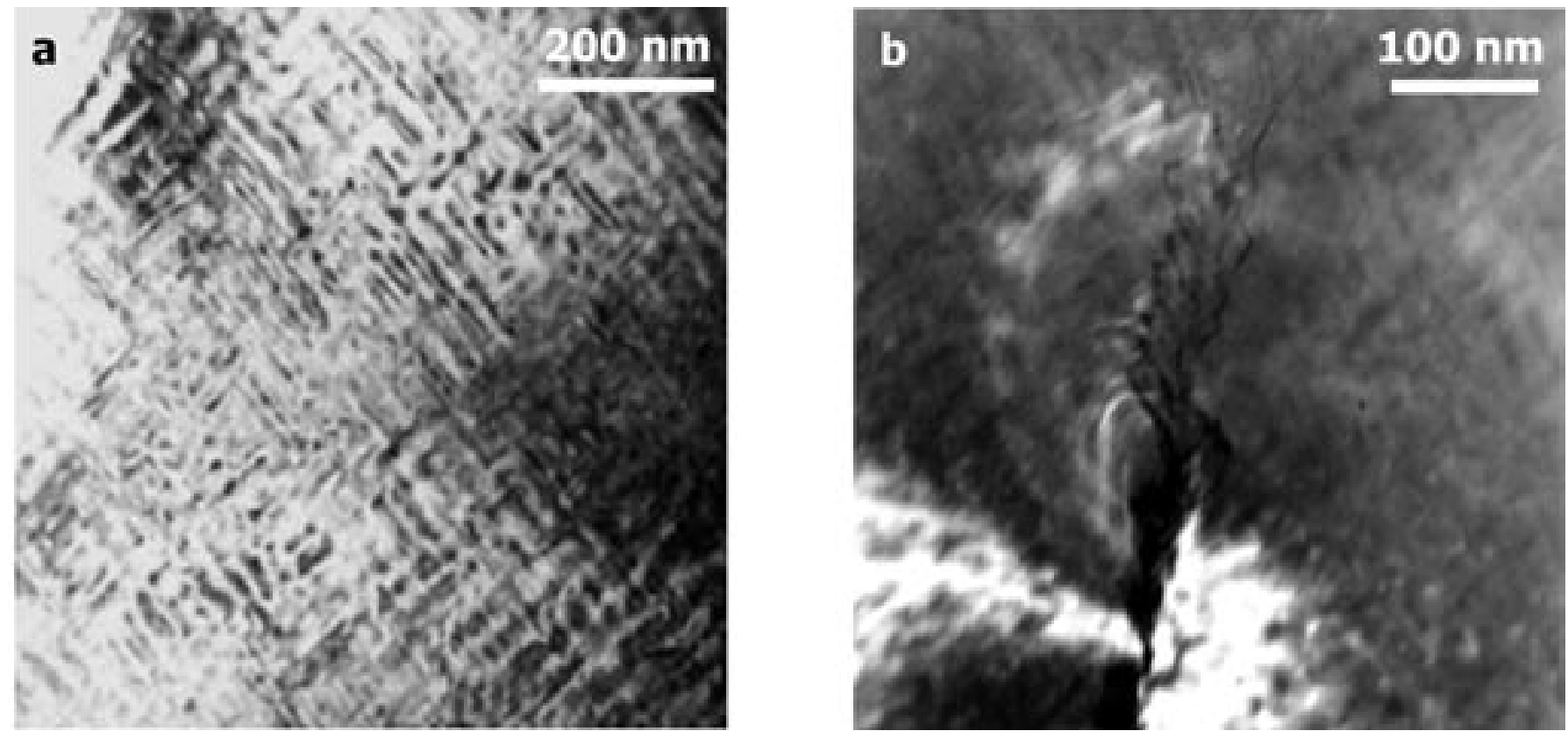

Fig. 5.- Imágenes de microscopía electrónica de transmisión (TEM) de la nanoestructura de las muestras estudiadas.

figura 3, al igual que el marcado como 4e, correspondiente al otro conjunto de líneas.

En esta última fibra es importante también destacar el interesante comportamiento que se observa en las zonas más alejadas del defecto. Con ese objeto, se obtuvo el detalle de la figura $4 \mathrm{~b}$, así como sus perfiles asociados. En él, se puede observar la universalidad de la estructura de valles y crestas, así como la aparición de unas llamativas estructuras ramificadas en la zona más alejada del defecto (perfil 4h), donde aparecen subgrupos de líneas que se cruzan a ángulos similares a los que formaban originalmente los dos grandes grupos de líneas que se observan en la figura 2a. Esto lleva a sugerir la existencia de ángulos preferentes para la propagación de fractura.
Si bien los resultados expuestos hasta ahora ya son de interés, debido a su carácter novedoso y por presentar un notable grado de sistematización, hay otro factor que no debe pasarse por alto, y es la similaridad existente entre las estructuras de fractura (típicamente micrométricas) y las de precipitados (de orden nanométrico). Un estudio de estas fibras mediante microscopía electrónica de transmisión (figura 5) revela una estructura de precipitados (figura 5a) muy similar a la textura observada luego en la superficie de fractura (comparar con la figura 1a) pero de un tamaño dos órdenes de magnitud menor. Este hecho, unido a la evidencia de que las grietas se propagan de forma más favorable a través de las fronteras entre los precipitados (figura 5b), es una fuerte prueba de que el comportamiento fractográfico de 
la circona-erbia depende íntimamente de sus precipitados. Es más, el estudio de la autoafinidad (repetición de un mismo patrón a diversas escalas) en las superficies de fractura, disciplina en plena expansión (7), podría abrir la puerta a la determinación de la nanoestructura de un material dado a partir del estudio de su comportamiento dentro del rango micrométrico mediante técnicas como LSCM.

\section{CONCLUSIONES}

Este trabajo demuestra que la microscopía óptica confocal de barrido láser es una herramienta de gran utilidad en estudios fractográficos. Esta técnica permite cuantificar la topología de las superficie de fractura, lo que no es posible mediante microscopía electrónica de barrido, llegando a observarse de forma más clara los detalles cuyo contraste está basado en una diferencia de altura.

La aplicación de esta técnica a fibras de $\mathrm{ZrO}_{2}-5 \%$ mol $\mathrm{Er}_{2} \mathrm{O}_{3^{\prime}}$ sometidas a tensión en condiciones de alta temperatura, ha permitido identificar en detalle sus características fractográficas, así como determinar las similitudes entre las distintas bandas formadas en el proceso de fractura y su relación con su estructura interna de precipitados.

\section{AGRADECIMIENTOS}

Investigación financiada por el Ministerio de Educación y Ciencia, proyecto MAT2003-06085. Las fibras de circona-erbia utilizadas fueron fabricadas en NASA Glenn Research Center por el Dr. Ali Sayir.

\section{BIBLIOGRAFÍA}

1. D. J. Draeger, E. D. Case. "Engineering the surface texture and shape of channels in ceramic substrates". Mat. Sci. Eng. B97 94-105 (2003).

2. J. J. Quispe Cancapa, A. R. de Arellano López, J. Martínez Fernández, A. Sayir. "Slow-Crack Growth in Chromia-Doped Sapphire". J. Eur. Ceram. Soc. 25 1259-1268 (2005).

3. J. J. Quispe Cancapa. “Propiedades mecánicas de fibras cerámicas monocristalinas fabricadas por solidificación direccional". Universidad de Sevilla, 2003 (tesis doctoral).

4. J. M. López-Cepero, J. J. Quispe Cancapa, A. Ramírez de Arellano López, J. Martínez Fernández. "Estudios fractográficos mediante microscopía óptica confocal”. Bol. Soc. Esp. Ceram. V. 44 [4] 231-238 (2005).

5. J. J. Quispe Cancapa, A. R. Pinto Gómez, J. Martínez Fernández, A. Sayir. "High Temperature Plastic Behavior Of Directionally Solidified $5 \mathrm{Mol} \%$ $\mathrm{Er}_{2} \mathrm{O}_{3}$ Doped $\mathrm{ZrO}_{2}$ Fibers And Bulk Crystals". Ceram. Sci. Eng. Proc. 23 [3] 673-678 (2002).

6. J. J. Quispe Cancapa, A. Ramirez de Arellano López, A. Sayir. "High Temperature Mechanical Properties of Directionally Solidified $\mathrm{Cr}^{+3}$ Sapphire Fibers". Ceram. Eng. Sci. Proc. 23 [3] 671-677 (2002).

7. M. Hinojosa Rivera, L. Chávez Guerrero. “Autoafinidad de superficies de fractura del vidrio". Ingenierías IV [13] 27-31 (2001).

Recibido: 09.01 .05

Aceptado: 22.08 .05 